\title{
Self-reported sleep disturbance and incidence of dementia in ageing men
}

\author{
Maria K Luojus, ${ }^{1}$ Soili M Lehto, ${ }^{2,3}$ Tommi Tolmunen, ${ }^{2,3}$ Anna-Katharine Brem, ${ }^{4,5,6}$ \\ Eija Lönnroos, ${ }^{1}$ Jussi Kauhanen ${ }^{1}$
}

- Additional material is published online only. To view please visit the journal online (http://dx.doi.org/10.1136/jech2016-207764).

${ }^{1}$ Institute of Public Health and Clinical Nutrition, University of Eastern Finland, Kuopio, Finland

${ }^{2}$ Institute of Clinical Medicine/ Psychiatry, University of Eastern Finland, Kuopio, Finland ${ }^{3}$ Department of Psychiatry, Kuopio University Hospital, Kuopio, Finland

${ }^{4}$ Max Planck Institute of Psychiatry, Munich, Germany ${ }^{5}$ Department of Experimental Psychology, University of Oxford, Oxford, UK

${ }^{6}$ Department of Neurology, Berenson-Allen Center for Noninvasive Brain Stimulation, Beth Israel Deaconess Medical Center, Harvard Medical School, Boston, Massachusetts, USA

\section{Correspondence to} Maria K Luojus, Institute of Public Health and Clinical Nutrition, University of Eastern Finland, P.O. Box 1627 Kuopio 70211, Finland; maria.luojus@uef.fi

Received 9 May 2016 Revised 30 September 2016 Accepted 1 October 2016
To cite: Luojus MK, Lehto SM, Tolmunen T, et al. J Epidemiol Community Health Published Online First: [please include Day Month Year] doi:10.1136/jech-2016207764

\section{ABSTRACT}

Background Sleep disturbance is suggested to contribute to the development of dementia. However, prospective longitudinal data from middle-aged populations are scarce.

Methods We investigated a population-based sample of 2386 men aged 42-62 years at baseline during 1984-1989. Participants having a history of mental illnesses, psychiatric medication, Parkinson's disease or dementia within 2 years after baseline $(n=296)$ were excluded. Difficulty falling asleep or maintaining sleep, sleep duration and daytime tiredness were enquired. Dementia diagnoses ( $n=287)$ between 1984 and 2014 were obtained through linkage with hospital discharge, national death and special reimbursement registers. Cox proportional hazards analyses were performed for all dementias, and separately for Alzheimer's disease $(n=234)$ and other phenotypes $(n=53)$. Additional analyses were performed on a subsample of an apolipoprotein E (APOE) genotype-tested population ( $n=1199)$.

Results The risk ratio for dementia was $1.58(95 \% \mathrm{Cl}$ 1.10 to 2.27) in men with frequent sleep disturbance after adjustments for age, examination year, elevated depressive symptoms, physical activity, alcohol consumption, cumulative smoking history, systolic blood pressure, body mass index, low-density lipoprotein and high-density lipoprotein cholesterol, high-sensitivity C reactive protein, cardiovascular disease history, education years and living alone. Daytime tiredness and sleep duration were not associated with dementia in adjusted analysis. In the $A P O E$ subsample, both $A P O E \varepsilon 4$ genotype and frequent sleep disturbance were associated with increased dementia risk, but in the interaction analysis they had no joint effect.

Conclusions Self-reported frequent sleep disturbance in middle-aged men may relate to the development of dementia in later life. Having an APOE $\varepsilon 4$ genotype did not affect the relationship.

\section{INTRODUCTION}

The likelihood of cognitive decline and alterations in the quality and duration of sleep increases with age. ${ }^{1}$ The prevalence of dementia doubles every 5 years after the age of $65 .^{2}$ Changes in sleep quality and duration both precede and co-occur with dementia. ${ }^{3}$ However, prospective sleep and dementia studies have mostly focused on participants older than 60 years with follow-up periods of 1-10 years. ${ }^{1}$ In the case of Alzheimer's disease (AD), the preclinical disease phase is already present $10-15$ years before the onset of clinical symptoms. ${ }^{4}$ During this early phase, soluble amyloid- $\beta$ becomes insoluble. ${ }^{5}$ Sleep-wake cycle alterations, poor sleep quality and awakenings during the night increase the concentration of soluble amyloid- $\beta$ due to enhanced cerebral synaptic activity. A bilateral relationship between amyloid- $\beta$ accumulation and sleep (sleep-wake cycle alterations ${ }^{6}$ and poor sleep ${ }^{4}{ }^{7}$ ) has been proposed in the pathophysiology of $\mathrm{AD}$.

$\mathrm{AD}$ accounts for $50-70 \%$ of all types of dementia. Other common types are vascular dementia $25 \%$, Lewy body dementias $15 \%{ }^{8}$ and frontotemporal dementia $10-20 \% .^{9}$ These dementia types share common risk factors such as a low educational level, sedentary lifestyle, smoking, alcoholism, hypertension, elevated inflammation (high-sensitivity C reactive protein (hs-CRP) $>0.3 \mathrm{mg} / \mathrm{L}),{ }^{10}$ diabetes, stroke and a history of cardiovascular diseases. ${ }^{211}$ Symptoms of depression and dementia are linked. Depression has been suggested to be the earliest manifestation of dementia before the appearance of cognitive impairment. ${ }^{12}$

The apolipoprotein $\mathrm{E}(A P O E)$ \&4 genotype is related to the development of vascular dementia and $\mathrm{AD} .{ }^{13} \mathrm{An}$ increased $\mathrm{AD}$ risk results from the contribution of APOE $\varepsilon 4$ to the density of neurofibrillary tangles and amyloid- $\beta$ accumulation. ${ }^{14}$ Obstructive sleep apnoea studies ${ }^{15}{ }^{16}$ have suggested that the APOE \&4 genotype modifies the relationship between sleep and cognitive impairment through unfavourable lipid metabolism. Based on observed neurofibrillary pathology, better sleep consolidation attenuates the relationship between the APOE $\varepsilon 4$ genotype and incident AD. ${ }^{17}$ Furthermore, the APOE $\varepsilon 4$ genotype is associated with an increased risk of insomnia. ${ }^{18}$

Self-reported short and long sleep, ${ }^{19}$ poor sleep quality, ${ }^{20}{ }^{21}$ awakenings after sleep onset ${ }^{22}$ and daytime sleepiness ${ }^{23}$ associate with cognitive impairment in the general population of older adults (55-82 years) without major depressive disorder, psychiatric medication, and prior neurodegenerative disease or obstructive sleep apnoea. Prospective studies have shown that difficulties falling asleep, nocturnal awakenings, short and long sleep duration, changes in sleep duration, poor sleep quality, daytime sleepiness and the use of hypnotics precede cognitive impairment and dementia. ${ }^{19}{ }^{24-33}$ To the best of our knowledge, prospective studies in participants who are still middle-aged at baseline are scarce. ${ }^{25}{ }^{26}$ In order to gain further information on midlife sleep disturbances that may precede dementia in later life, we investigated the associations of self-reported sleep duration and quality with incident physiciandiagnosed dementia in a prospective follow-up setting among 2386 middle-aged men from Eastern 
Finland. Finally, since the APOE $\varepsilon 4$ genotype has been found to be connected to poor sleep and dementia, ${ }^{17}{ }^{18}$ we assessed whether the association between sleep disturbance and incident dementia is moderated by the APOE $\varepsilon 4$ genotype.

\section{METHODS}

\section{Study population}

Participants in the Kuopio Ischemic Heart Disease Study (KIHD) were randomly selected from the general population of Kuopio and surrounding areas in Eastern Finland. ${ }^{34}$ Baseline data were collected between the years 1984 and 1989 from 2682 men $(82.9 \%$ participation rate of the invited 3235 eligible men). The men were aged 42-62 years at the time of baseline examinations (mean age $53 \pm 5.2$ years). The mean follow-up time for incident dementia was $21.9 \pm 7.9$ years. Participants with a history of mental illnesses, use of psychiatric medication (hypnotics, sedatives or medication for depression) at baseline, or a diagnosis of Parkinson's disease or dementia within 2 years after the baseline $(n=296)$ were excluded from the study, leaving a total of 2386 respondents. Further exclusions for hospital discharge register-based diagnoses of obstructive sleep apnoea, or myocardial infarction, atrial fibrillation and heart failure were conducted for supplementary analyses. Information on APOE genotypes was available for 1199 participants. Additional statistical analyses were performed on the APOE sample. The guidelines laid down in the Declaration of Helsinki were taken into account, and all study procedures were approved by the Committee on Research Ethics of the University of Kuopio. Written informed consent was obtained from all participants.

\section{Outcome}

Three national health registers were used to identify incident cases of dementia in the KIHD cohort. The International Classification of Diseases (ICD)-8 code 290, ICD-9 codes 4378A and 290, and ICD-10 codes F00, F01, F02, F03, G30 and G31 were recorded from the hospital discharge register ${ }^{35}$ and the national death register. ${ }^{36}$ The search covered the period from the beginning of 1984 to the end of 2014. Since 1999, a few years after the first drugs for the symptomatic treatment of $\mathrm{AD}$ were launched, diagnosis of $\mathrm{AD}$ has been recorded in the Special Reimbursement Register (SRR) maintained by the Social Insurance Institution (SII) of Finland. The SRR is often used as a clinical epidemiology data source for studies on specific chronic conditions, ${ }^{37} 38$ including the prevalence and incidence of $\mathrm{AD} .^{3940}$ To receive a special reimbursement right, the patient has to be examined, diagnosed and given a certificate by a medical physician. For a diagnosis of $\mathrm{AD}$ to be verified and recorded in the SRR, the following conditions are required to be met: the person has (1) symptoms consistent with $\mathrm{AD},(2)$ experienced a decrease in social capacity over a period of at least 3 months, (3) received a CT or MRI scan, (4) had possible alternative diagnoses excluded and (5) received confirmation of the diagnosis by a registered neurologist or geriatrician. Each medical certificate is then assessed by the SII to ensure that a patient meets the diagnostic criteria of the Diagnostic and Statistical Manual Version IV (DSM-IV) and NINCDS-ADRDA for AD. ${ }^{41-43}$ Persons with mixed-type dementia, that is, AD/vascular and $\mathrm{AD} /$ Lewy body, are also recorded. $\mathrm{AD}$ is the most common cause of dementia, and linkage to the SRR proved to be the strongest method to identify cases of AD-type dementia in the KIHD cohort. The SRR data covered the period from the beginning of 1999 to the end of 2014 .

\section{Baseline measures}

\section{Sleep}

Participants completed self-administered questionnaires that were analysed by an interviewer. Sleep disturbance was enquired with the question: 'How often do you have difficulties falling asleep or maintaining sleep?'. Response alternatives were: 'never or seldom', 'occasionally' and 'often'. Information on sleeping hours was obtained with the question: 'How many hours do you usually sleep at night?' Response alternatives were: $\leq 6,6.5$, $7,7.5,8,8.5,9,9.5$ and $\geq 10$ hours. Three categories were constructed: $\leq 6.5,7-8$ and $\geq 8.5$ hours. Daytime tiredness was enquired with the question: 'Do you get tired easily?' with the response alternatives 'yes' or 'no'.

\section{Health and sociodemographic background}

Depressive symptoms were assessed with the 18-item Human Population Laboratory (HPL) depression scale. ${ }^{44}$ The items evaluate symptoms such as mood disturbance, negative selfconcept, energy loss, poor appetite, concentration difficulties and psychomotor agitation. The HPL depression scale score is generated by assigning one point for each true or false answer indicative of depression (range 0-18). The insomnia item of this scale was excluded. A cut-off point of $\geq 5$ is used to define elevated depressive symptoms. ${ }^{44}$

To assess physical activity, the 12-Month Physical Activity questionnaire $^{45}$ was administered. This checklist includes the most common physical activities of Finnish middle-aged men (walking, jogging, skiing, bicycling, swimming and games involving physical activity). The participants were asked to record the frequency, average duration and intensity. The energy expenditure from physical activity was expressed as kcal/day.

Alcohol consumption (g/week) was estimated with a structured quantity-frequency method using the Nordic Alcohol Consumption Inventory for drinking behaviour over the previous 12 months. ${ }^{46}$ Cumulative smoking history (pack-years) was estimated as the product of years smoked and the number of tobacco products smoked daily at the time of examination.

Blood pressure was measured with a random-zero mercury sphygmomanometer. The mean value $(\mathrm{mm} \mathrm{Hg})$ of six measurements (three while supine, two while sitting and one while standing) was used. Body mass index (BMI) was computed as the ratio of weight (kilograms) to the square of height (metres). In brief, venous blood sampling was performed between 8:00 and 10:00. Participants were instructed to abstain from alcohol use for 3 days and from smoking and eating for 12 hours. Serum low-density lipoprotein (LDL) cholesterol was precipitated by using polyvinyl sulfate (Boehringer Mannheim, Germany) and calculated as the difference between total and supernatant cholesterol. The serum high-density lipoprotein (HDL) cholesterol concentration was determined after precipitation with magnesium chloride dextran sulfate. hs-CRP was measured with an immunometric assay, the Immulite High Sensitivity CRP Assay (Diagnostic Products Corporation, Los Angeles, California, USA), which was standardised against the WHO International Reference Standard for CRP immunoassay $85 / 506$. At the level of $3.2 \mathrm{mg} / \mathrm{L}$, the within-run coefficient of variation was $2.8 \%$ and the total coefficient of variation was $3.1 \%$.

A positive cardiovascular disease history was determined based on the following criteria: at least one physician-diagnosed cardiovascular disease (ie, myocardial infarction, angina pectoris, other coronary disease, cardiomyopathy or cardiac insufficiency) and/or nitrate use at least once per week. Angina 
pectoris was assessed according to the WHO angina pectoris questionnaire (the Rose Angina Questionnaire), a validated instrument to assess symptoms of typical angina pectoris in general populations. ${ }^{47}$ Mental disease history was enquired with the questions: 'Have you ever had physician-diagnosed mental illness?' ('yes' or 'no'), and 'Have you ever had physiciandiagnosed severe psychiatric disease?' ('yes' or 'no').

Information on APOE genotypes was available for 1199 men. The APOE genotypes APOE2 (cys112, cys158), APOE3 (cys112, $\arg 158)$ and APOE4 (arg112, $\arg 158)$ were determined by using the PCR-restriction fragment length polymorphism method and HhaI digestion, as described by Tsukamoto et al. ${ }^{48}$ The APOE genotype statuses were categorised as follows: (1) homozygotic and heterozygotic genotypes of APOE2 and APOE3 (22,23,33); (2) heterozygotic genotypes of APOE4 $(42,43)$; and (3) homozygotic genotype of APOE4 (44). Categories (2) and (3) were combined for interaction analyses. In addition to health characteristics, participants were asked about use of medication, total years of education and marital status (married or living with spouse vs living alone).

\section{Statistics}

Kaplan-Meier survival analysis (Mantel-Cox log-rank test) was applied to assess the differences in the incidence of any type of dementia, $\mathrm{AD}$ and other phenotypes among the different sleep variable categories. To compute adjusted risk ratios and CIs, Cox proportional hazards models were used. Covariates were selected based on previous studies. ${ }^{1}$ The differences between the baseline characteristics in sleep disturbance categories were explored with the Kruskal-Wallis test due to non-normal distributions of the variables. The $\chi^{2}$ test was used for the categorical variables. For the Cox proportional hazards models, we first built a model ${ }^{\mathrm{a}}$ that was adjusted for age and examination years. Model $^{\mathrm{a}}$ was then supplemented with HPL scale scores $\geq 5$ to form model ${ }^{\mathrm{b}}$. Model ${ }^{\mathrm{b}}$ was further adjusted for: (1) physical activity (kcal/day), alcohol consumption (g/week) and cumulative smoking history (pack-year; model $^{\mathrm{c}}$ ); or (2) systolic blood pressure $(\mathrm{mm} \mathrm{Hg}), \mathrm{BMI}, \mathrm{LDL}$ and HDL cholesterol $(\mathrm{mmol} / \mathrm{L})$, hs-CRP $(\mathrm{mg} / \mathrm{L})$, and cardiovascular disease history $\left(\right.$ model $\left.^{\mathrm{d}}\right)$; or (3) education years and marital status $\left(\operatorname{model}^{\mathrm{e}}\right)$. Finally, we built model ${ }^{\mathrm{f}}$, which included all covariates from models ${ }^{\mathrm{a}-\mathrm{e}}$. Additional analysis of the interactive effects of sleep disturbance and $A P O E$ genotypes on dementia risk was carried out in the $A P O E$ genotype-tested population. The interaction variable was formed by multiplying the sleep disturbance and APOE genotype variables. Data were analysed using SPSS Statistics V.21.0 and V.23.0 (IBM Corp, SPSS Statistics).

\section{RESULTS}

\section{Self-reported sleep disturbances related to dementia}

On average, men received dementia diagnoses between 67 and 79 years of age. In the Kaplan-Meier survival analysis (using the Mantel-Cox log-rank test), sleep disturbance $(p=0.002)$ and daytime tiredness $(p<0.019)$ were associated with the risk of dementia, whereas sleep duration $(p=0.554)$ was not. A total of $149(25.9 \%)$ men who suffered from daytime tiredness reported frequent sleep disturbance, whereas 299 (51.9\%) men reported occasionally disturbed sleep.

In the adjusted Cox proportional hazards analysis, neither sleep duration (see online supplementary table S1) nor selfreported daytime tiredness (see online supplementary table S2) was significantly related to dementia. Therefore, further analyses were conducted for self-reported sleep disturbance only.

\section{Multivariate analyses for self-reported sleep disturbance and incident dementia}

Table 1 displays baseline health and sociodemographic characteristics according to the self-reported sleep disturbance

Table 1 Baseline characteristics according to self-reported sleep disturbance in ageing men $(n=2386)$

\begin{tabular}{|c|c|c|c|c|c|}
\hline & \multicolumn{3}{|l|}{ Sleep disturbance } & \multirow[b]{2}{*}{ Test statistic } & \multirow[b]{2}{*}{$p$ Value } \\
\hline & $\begin{array}{l}\text { Never/seldom } \\
n=799\end{array}$ & $\begin{array}{l}\text { Occasionally } \\
n=1258\end{array}$ & $\begin{array}{l}\text { Often } \\
n=329\end{array}$ & & \\
\hline Age years & $54.3(48.5-54.5)$ & $54.3(54.1-54.6)$ & $54.4(54.2-54.8)$ & $15.4^{*}$ & $<0.001^{*}$ \\
\hline Follow-up years for dementia & $25.9(20.1-27.8)$ & $25.3(17.3-27.7)$ & $23.6(12.1-27.2)$ & $21.7^{*}$ & $<0.001$ * \\
\hline HPL scale scores $\geq 5$ & $29(3.6)$ & $86(6.9)$ & $71(21.6)$ & $107.9+$ & $<0.001 \dagger$ \\
\hline PA (kcal/day) & $84.1(29.8-189.1)$ & $83.8(30.1-185.1)$ & $77.1(26.5-207.2)$ & $0.1^{*}$ & $0.953^{*}$ \\
\hline Alcohol (g/week) & $26.2(6.0-77.3)$ & $33.1(6.4-93.7)$ & $44.0(7.0-136.6)$ & $16.8^{*}$ & $<0.001^{*}$ \\
\hline \multicolumn{6}{|l|}{ Smoking (pack-years) (\%) } \\
\hline 0 & 565 (72.6) & $877(71.3)$ & $214(66.7)$ & $10.01 \dagger$ & $0.124 \dagger$ \\
\hline $1-500$ & $102(13.1)$ & $158(12.8)$ & $47(14.6)$ & & \\
\hline 501-999 & $94(12.1)$ & $161(13.1)$ & $43(13.4)$ & & \\
\hline$>1000$ & $17(2.2)$ & $34(2.8)$ & $17(5.3)$ & & \\
\hline Systolic BP (mm Hg) & 131.3 (122.7-142.7) & $132.0(122.7-144.3)$ & $133.3(122.8-144.0)$ & $1.6^{*}$ & $0.439 *$ \\
\hline BMI $\left(k g / m^{2}\right)$ & $26.2(24.4-28.6)$ & $26.6(24.6-29.1)$ & $26.3(24.2-28.8)$ & $3.6^{*}$ & $0.167^{*}$ \\
\hline LDL cholesterol (mmol/L) & $4.0(3.3-4.7)$ & $4.0(3.4-4.7)$ & $3.9(3.4-4.6)$ & $0.2^{*}$ & $0.974^{*}$ \\
\hline HDL cholesterol (mmol/L) & $1.3(1.1-1.5)$ & $1.3(1.1-1.5)$ & $1.2(1.1-11.5)$ & $0.1^{*}$ & $0.974^{*}$ \\
\hline Hs-CRP (mg/L) & $1.3(0.7-2.4)$ & $1.3(0.7-2.4)$ & $1.3(0.7-2.7)$ & $1.0^{*}$ & $0.616^{*}$ \\
\hline CVD history (\%) & $128(16.0)$ & $246(19.6)$ & $89(27.1)$ & $18.2 \dagger$ & $<0.001 \dagger$ \\
\hline Education (years) & $8.0(6.0-10.0)$ & $8.0(6.0-10.0)$ & $8.0(6.0-9.0)$ & $16.6^{*}$ & $<0.001^{*}$ \\
\hline Living alone (\%) & $43(5.4)$ & $81(6.4)$ & $20(6.1)$ & $1.0 t$ & $0.615 t$ \\
\hline
\end{tabular}


categories. Men reporting frequent sleep disturbance had $\sim 1.5$-fold increased risk for any type of incident dementia (table 2 ) in the fully adjusted Cox analysis $\left(\operatorname{model}^{\mathrm{f}}\right)$. The association was slightly weaker with $\mathrm{AD}$ as an outcome, and the full model failed to reach statistical significance. The observed relationship was similar with other dementia phenotypes, but due to low numbers it was not statistically significant (table 2). After exclusion of men who received a diagnosis of obstructive sleep apnoea during the 1984-2014 follow-up, any type of dementia and $\mathrm{AD}$ was associated with incident dementia in fully adjusted Cox analyses (see online supplementary table S3). When men having myocardial infarction, atrial fibrillation or heart failure during the 1984-2013 follow-up were excluded from the study population, a statistically significant relationship was observed for any type of dementia (see online supplementary table S4).

In the APOE subsample, self-reported sleep disturbance, and homozygotic and heterozygotic $A P O E \& 4$ genotypes were related to an increased dementia risk (table 3 ) after adjusting for age, examination year and HPL scale scores $\geq 5$. The selfreported sleep disturbance and $A P O E \varepsilon 4$ genotype had no significant interaction with respect to increased risk of dementia.

\section{DISCUSSION}

\section{Summary of main findings}

Middle-aged men who reported frequent sleep disturbance had $\sim 1.5$-fold increased risk of physician-diagnosed dementia in later life. These observations remained significant regardless of adjustments for age, elevated depressive symptoms, physical activity, alcohol consumption, cumulative smoking history, systolic blood pressure, BMI, LDL and HDL cholesterol, hs-CRP, cardiovascular disease history, education years and living alone. In the subsample of the APOE genotype-tested population, selfreported sleep disturbance and the $A P O E \varepsilon 4$ genotype were related to an increased dementia risk, but there was no significant interaction between sleep disturbance and genotype.

\section{Comparison with previous literature}

Our observation of an increased risk of incident dementia in middle-aged men who reported frequent sleep disturbance may support the association between self-reported sleep disturbance and any type of dementia. ${ }^{1}$ Since most patients with dementia were diagnosed with $\mathrm{AD}$, the observations with all types of dementias and $\mathrm{AD}$ were in line, although the association with $\mathrm{AD}$ was slightly weaker. The estimated risk of other phenotypes of dementia was of the same magnitude, or even higher, but due to low number of outcomes the study did not have sufficient statistical power for this analysis.

The observed relationship with dementia is in line with findings reported by Jelicic et $a l,{ }^{26}$ suggesting that subjectively experienced sleep disturbance in middle age increases the risk of cognitive decline. The study ${ }^{26}$ was conducted among 402 women and 436 men who were aged >50 years at the study baseline (average age 63.3 \pm 9.1 years). Sleep disturbance was enquired with the Symptom Checklist-90. Mini-Mental State Examination was used to determine cognitive impairment both at baseline and at 3-year follow-up. The authors concluded that depressive symptoms mediated the relationship between sleep disturbance and cognitive decline, because the association became non-significant after controlling for depressive symptoms. Our data suggest that depressive symptoms do not fully explain the associations between sleep disturbance and dementia, because adjusting the observed association for elevated depressive symptoms did not change the results. However, owing to the multifactorial nature of poor sleep, sleep disturbance can be a marker for health-related factors that increase the risk of dementia. ${ }^{49}$

Several physiological pathways underlie the link between sleep disturbance and the development of dementia. Chronic insomnia reduces the brain volume. ${ }^{1}$ However, the results from structural brain imaging studies among individuals suffering from insomnia are somewhat conflicting. A reduction in the

Table 2 Self-reported sleep disturbance and risk ratios (with 95\% Cl) for incident dementia in ageing men ( $\mathrm{n}=2386$ )

\begin{tabular}{|c|c|c|c|c|c|c|}
\hline Sleep disturbance & Model $^{\mathrm{a}}$ & Model $^{b}$ & Model $^{\mathrm{c}}$ & Model $^{d}$ & Model $^{\mathrm{e}}$ & Model $^{f}$ \\
\hline & \multicolumn{6}{|c|}{ Any type of dementia $n=287$} \\
\hline $\begin{array}{l}\text { Never/seldom } \\
\mathrm{n}=799\end{array}$ & ref & ref & ref & ref & ref & ref \\
\hline $\begin{array}{l}\text { Occasionally } \\
\mathrm{n}=1258\end{array}$ & $1.08(0.83$ to 1.41$)$ & $1.07(0.82$ to 1.40$)$ & 1.03 (0.79 to 1.35$)$ & 1.09 (0.83 to 1.43$)$ & 1.06 (0.81 to 1.38$)$ & $1.04(0.79$ to 1.36$)$ \\
\hline \multirow[t]{2}{*}{$\begin{array}{l}\text { Often } \\
n=329\end{array}$} & 1.71 (1.21 to 2.41$)$ & 1.68 (1.18 to 2.40$)$ & 1.55 (1.08 to 2.21$)$ & 1.71 (1.20 to 2.44$)$ & 1.67 (1.172 to 2.38$)$ & 1.58 (1.10 to 2.27$)$ \\
\hline & \multicolumn{6}{|c|}{ Alzheimer's disease $n=234$} \\
\hline $\begin{array}{l}\text { Never/seldom } \\
\mathrm{n}=799\end{array}$ & ref & ref & ref & ref & ref & ref \\
\hline $\begin{array}{l}\text { Occasionally } \\
n=1258\end{array}$ & 1.07 (0.80 to 1.42$)$ & $1.06(0.79$ to 1.42$)$ & 1.03 (0.77 to 1.37 ) & $1.08(0.80$ to 1.46$)$ & 1.05 (0.78 to 1.40$)$ & $1.04(0.77$ to 1.40$)$ \\
\hline \multirow{2}{*}{$\begin{array}{l}\text { Often } \\
n=329\end{array}$} & 1.52 (1.03 to 2.23$)$ & 1.52 (1.02 to 2.25$)$ & 1.42 (0.95 to 2.12 ) & 1.56 (1.05 to 2.33$)$ & 1.50 (1.01 to 2.23$)$ & 1.46 (0.97 to 2.20$)$ \\
\hline & \multicolumn{6}{|c|}{ Dementia excluding Alzheimer's disease $n=53$} \\
\hline $\begin{array}{l}\text { Never/seldom } \\
\mathrm{n}=799\end{array}$ & ref & ref & ref & ref & ref & ref \\
\hline $\begin{array}{l}\text { Occasionally } \\
\mathrm{n}=1258\end{array}$ & $1.18(0.61$ to 2.25$)$ & $1.14(0.59$ to 2.19$)$ & 1.10 (0.57 to 2.13 ) & 1.11 (0.57 to 2.13 ) & 1.13 (0.59 to 2.18 ) & $1.08(0.56$ to 2.10$)$ \\
\hline $\begin{array}{l}\text { Often } \\
n=329\end{array}$ & 2.55 (1.20 to 5.42$)$ & 2.35 (1.07 to 5.15$)$ & 1.99 (0.89 to 4.44$)$ & 2.14 (0.97 to 4.71$)$ & 2.33 (1.06 to 5.13$)$ & 1.93 (0.87 to 4.29$)$ \\
\hline
\end{tabular}

Model $^{\mathrm{a}}$ adjusted for age and examination year; model ${ }^{\mathrm{b}}$ further adjusted for Human Population Laboratory depression scale scores $\geq 5$; model ${ }^{\mathrm{c}}$ further adjusted for physical activity (kcal/ day), alcohol consumption (g/week) and cumulative smoking history (pack-years); model ${ }^{\mathrm{d}}$ : model ${ }^{\mathrm{b}}$ further adjusted for systolic blood pressure ( $\mathrm{mm} \mathrm{Hg}$ ), body mass index $\left(\mathrm{kg} / \mathrm{m}^{2}\right)$, low-density and high-density lipoprotein cholesterol (mmol/L), high-sensitivity C reactive protein (mg/L) and cardiovascular disease history; model ${ }^{\mathrm{e}}$ : model ${ }^{\mathrm{b}}$ further adjusted for education years and living alone; modelf: includes model ${ }^{\mathrm{a}-\mathrm{e}}$ covariates. 
Table 3 The RRs with $95 \% \mathrm{Cl}$ for incident dementias according to APOE genotypes and self-reported sleep disturbance in ageing men $(n=1199)$

\begin{tabular}{|c|c|c|c|c|}
\hline & \multicolumn{2}{|c|}{ Any type of dementia $n=145$} & \multicolumn{2}{|c|}{ Alzheimer's disease $n=123$} \\
\hline & $n(\%)$ & $\operatorname{RR}(95 \% \mathrm{CI})^{\mathbf{a}}$ & n (\%) & $\mathbf{R R}(95 \% \mathrm{CI})^{\mathbf{a}}$ \\
\hline \multicolumn{5}{|l|}{ APOE genotypes } \\
\hline $22,23,33 n=792$ & $74(9.3)$ & ref & $56(7.1)$ & ref \\
\hline $42,43 n=365$ & $51(14.0)$ & 1.60 (1.11 to 2.29$)$ & $48(13.2)$ & 1.96 (1.33 to 2.89$)$ \\
\hline $44 n=42$ & $20(47.6)$ & 4.74 (2.86 to 7.84$)$ & $19(45.2)$ & 5.80 (3.42 to 9.85$)$ \\
\hline \multicolumn{5}{|l|}{ Sleep disturbance } \\
\hline Never/seldom $n=437$ & $46(10.5)$ & ref & $39(8.9)$ & ref \\
\hline Occasionally $n=614$ & $78(12.7)$ & $1.24(0.86$ to 1.79$)$ & $68(11.1)$ & 1.30 (0.88 to 1.93$)$ \\
\hline \multirow[t]{2}{*}{ Often $n=148$} & $21(14.2)$ & 1.71 (1.01 to 2.92$)$ & $16(10.8)$ & 1.60 (0.88 to 2.89$)$ \\
\hline & & $\operatorname{RR}(95 \% \mathrm{Cl})^{\mathbf{b}}$ & & $\operatorname{RR}(95 \% \mathrm{Cl})^{\mathrm{c}}$ \\
\hline \multicolumn{5}{|l|}{ Interaction } \\
\hline Sleep disturbance never/seldom $\times 22,23,33$ & & ref. & & ref. \\
\hline Sleep disturbance occasionally $\times 42,43,44$ & & $1.73(0.83$ to 3.60$)$ & & $1.60(0.72$ to 3.55$)$ \\
\hline Sleep disturbance often $\times 42,43,44$ & & $0.60(0.20$ to 1.74$)$ & & $0.54(0.17$ to 1.76$)$ \\
\hline \multicolumn{5}{|c|}{ 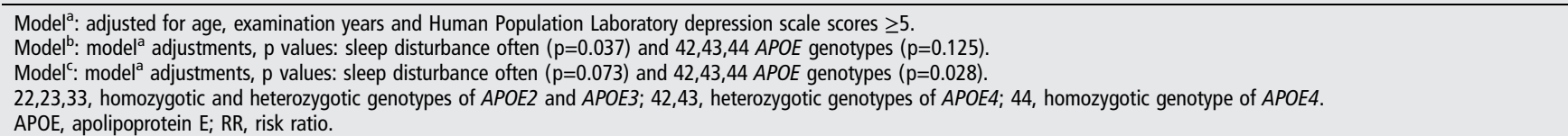 } \\
\hline
\end{tabular}

hippocampal volume $e^{50} 51$ and orbitofrontal and parietal grey matter $^{52}$ has been observed, or brain volume changes have not been found. ${ }^{53} 54$ Poor sleep and sleep loss are proposed to induce neuroinflammation ${ }^{55}$ and disruptions in the production and development of new neurons. ${ }^{56}$ Furthermore, accumulation of amyloid- $\beta$ may disrupt the sleep-wake cycle. As the amyloid- $\beta$ concentration increases, more sleep-wake cycle alterations occur during the development of $\mathrm{AD}^{6}$

Contrary to previous cross-sectional ${ }^{23}$ and prospective ${ }^{31}$ studies, we did not observe a statistically significant relationship between daytime tiredness and dementia in the adjusted multivariate analysis. Daytime tiredness can occur as a primary condition, ${ }^{57}$ or secondarily due to periodic limb movement disorder, insomnia, depression, and cardiovascular or pulmonary disease. 15859

A recently conducted meta-analysis ${ }^{19}$ concerning self-reported sleep duration and cognitive performance established that both short and long sleep in older age ( $>55$ years) have been associated with cognitive decline in cross-sectional and prospective studies. Contrary to previous knowledge, we did not observe a statistically significant relationship between self-reported sleep duration and incident dementia.

Previous data on the interaction between the APOE $\varepsilon 4$ genotype and sleep regarding an increased dementia risk are scarce. Lim et $a l^{17}$ analysed 10-day actigraphic recordings among 698 community-dwelling older adults (mean age 81.7 years). The authors hypothesised that the risk of incident $\mathrm{AD}$ varies depending on the interaction between the APOE $\varepsilon 4$ genotype and sleep consolidation. The results from the interaction analysis revealed that each $1 \mathrm{SD}$ increase in sleep consolidation attenuated the effect of the APOE $\varepsilon 4$ genotype on the $\mathrm{AD}$ risk by $\sim 50 \%$ during the 6-year follow-up period. The authors concluded that better sleep consolidation attenuated the effect of the APOE $\varepsilon 4$ genotype on the risk of incident $\mathrm{AD}$ via observed amyloid- $\beta$ pathological effects on the density of neurofibrillary tangles.

The prevalence of the homozygotic APOE \&4 genotype among those with $\mathrm{AD}$ is high, being $\sim 14.1 \%$ in Northern
Europe. ${ }^{60}$ We observed a slightly higher prevalence of $16.7 \%$ among our population with AD. Nevertheless, there is a need for further research on the association between self-reported sleep disturbance in middle age and incident dementia in larger samples within prospective study settings.

\section{Strengths and limitations}

Our study comprised a regionally representative populationbased sample of ageing men with a high participation rate. The linkage with three national healthcare registers provided us detailed and clinically confirmed information on incident dementia in the study cohort during the whole follow-up. We were able to take into account several potential confounders, such as elevated depressive symptoms and cardiovascular factors, which contribute to dementia morbidity. To further avoid potentially confounding effects, we excluded participants using psychotropic medication or with a diagnosis of Parkinson's disease.

The following limitations need to be considered when interpreting the findings: (1) the results may not be generalisable to women and younger men; (2) self-reported sleep duration and quality variables may overestimate or underestimate sleep disturbance. Validated scales measuring sleep disturbances could have provided more comprehensive information on the different aspects of sleep; (3) we did not obtain a screening measure of cognitive decline (Mini-Mental State Examination or another comparable test), and we were therefore unable to specifically categorise mild, moderate or severe cognitive impairment; (4) medical records for psychotropic or hypnotic medication during the follow-up were not available.

\section{CONCLUSIONS}

Self-reported frequent insomnia in middle-aged men is associated with $\sim 1.5$-fold increased risk of dementia in later life. However, the increased risk was not observed among APOE $\varepsilon 4$ allele carriers. Our observation emphasises the role of poor sleep quality in the development of dementia. 


\section{What is already known on this subject}

- Sleep disturbance both precedes and co-occurs with dementia.

- The preclinical disease phase of Alzheimer's disease begins 10-15 years before the onset of clinical symptoms.

- The APOE \&4 genotype increases the dementia risk and modifies the relationship between obstructive sleep apnoea and dementia.

\section{What this study adds}

Our data suggest that frequent sleep disturbance in middle age (ie, 42-62 years of age) increases the risk of dementia in later life.

Acknowledgements The authors thank the former personnel of the Research Institute of Public Health for their valuable work in the KIHD data gathering, and Kimmo Ronkainen, MSc, for data management.

Contributors MKL planned and conducted the study (including statistical analyses), and wrote the manuscript. SML, TT, A-KB and JK planned the study, revised the manuscript and participated in the statistical analyses. EL planned the revised manuscript and participated in the statistical analyses.

Funding MKL was supported by a grant from the Juha Vainio Foundation. The work of SML was supported by the Paulo Foundation.

Competing interests None declared.

Patient consent Obtained.

Ethics approval Committee on Research Ethics of the University of Kuopio.

Provenance and peer review Not commissioned; externally peer reviewed.

\section{REFERENCES}

1 Yaffe K, Falvey CM, Hoang T. Connections between sleep and cognition in older adults. Lancet Neurol 2014;13:1017-28.

2 Hugo J, Ganguli M. Dementia and cognitive impairment: epidemiology, diagnosis, and treatment. Clin Geriatr Med 2014:30:421-42.

3 Guarnieri B, Sorbi S. Sleep and cognitive decline: a strong bidirectional relationship. It is time for specific recommendations on routine assessment and the management of sleep disorders in patients with mild cognitive impairment and dementia. Eur Neurol 2015;74:43-8.

4 Ju YE, Lucey BP, Holtzman DM. Sleep and Alzheimer disease pathology-a bidirectional relationship. Nat Rev Neurol 2014;10:115-19.

5 Sperling RA, Aisen PS, Beckett LA, et al. Toward defining the preclinical stages of Alzheimer's disease: recommendations from the National Institute on Aging-Alzheimer's Association workgroups on diagnostic guidelines for Alzheimer's disease. Alzheimers Dement 2011;7:280-92.

6 Lim MM, Gerstner JR, Holtzman DM. The sleep-wake cycle and Alzheimer's disease: what do we know? Neurodegener Dis Manag 2014;4:351-62.

7 Ju YE, McLeland JS, Toedebusch CD, et al. Sleep quality and preclinical Alzheimer disease. JAMA Neurol 2013;70:587-93.

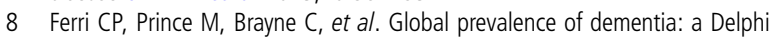
consensus study. Lancet 2005;366:2112-17.

9 Luukkainen L, Bloigu R, Moilanen V, et al. Epidemiology of frontotemporal lobar degeneration in northern Finland. Dement Geriatr Cogn Dis Extra 2015;5:435-41.

10 Schmidt R, Schmidt H, Curb JD, et al. Early inflammation and dementia: a 25-year follow-up of the Honolulu-Asia Aging Study. Ann Neurol 2002;52:168-74.

11 Duarte JM. Metabolic alterations associated to brain dysfunction in diabetes. Aging Dis 2015:6:304-21.

12 Panza F, Frisardi V, Capurso C, et al. Late-life depression, mild cognitive impairment, and dementia: possible continuum? Am J Geriatr Psychiatry 2010:18:98-116.

13 Giau VV, Bagyinszky E, An SS, et al. Role of apolipoprotein E in neurodegenerative diseases. Neuropsychiatr Dis Treat 2015:11:1723-37.
14 Shanthi KB, Krishnan S, Rani P. A systematic review and meta-analysis of plasma amyloid 1-42 and tau as biomarkers for Alzheimer's disease. SAGE Open Med 2015;3:2050312115598250

15 Nikodemova M, Finn L, Mignot E, et al. Association of sleep disordered breathing and cognitive deficit in APOE $\varepsilon 4$ carriers. Sleep 2013;36:873-80.

16 Spira AP, Blackwell T, Stone KL, et al. Sleep-disordered breathing and cognition in older women. J Am Geriatr Soc 2008;56:45-50.

17 Lim AS, Yu L, Kowgier M, et al. Modification of the relationship of the apolipoprotein $\mathrm{E} \varepsilon 4$ allele to the risk of Alzheimer disease and neurofibrillary tangle density by sleep. JAMA Neurol 2013;70:1544-51.

18 Wang CC, Lung FW. The role of PGC-1 and Apoe4 in insomnia. Psychiatr Genet 2012:22:82-7.

19 Lo JC, Groeger JA, Cheng GH, et al. Self-reported sleep duration and cognitive performance in older adults: a systematic review and meta-analysis. Sleep Med 2016:17:87-98.

20 Nebes RD, Buysse DJ, Halligan EM, et al. Self-reported sleep quality predicts poor cognitive performance in healthy older adults. J Gerontol B Psychol Sci Soc 2009;64:180-7

21 Niu J, Han H, Wang Y, et al. Sleep quality and cognitive decline in a community of older adults in Daqing City, China. Sleep Med 2016;17:69-74.

22 Blackwell T, Yaffe K, Ancoli-Israel S, et al. Association of sleep characteristics and cognition in older community-dwelling men: the MrOS sleep study. Sleep 2011;34:1347-56

23 Merlino G, Piani A, Gigli GL, et al. Daytime sleepiness is associated with dementia and cognitive decline in older Italian adults: a population-based study. Sleep Med 2010;11:372-7.

24 Potvin 0 , Lorrain $\mathrm{D}$, Forget $\mathrm{H}$, et al. Sleep quality and 1-year incident cognitive impairment in community-dwelling older adults. Sleep 2012;35:491-9.

25 Ferrie JE, Shipley MJ, Akbaraly TN, et al. Change in sleep duration and cognitive function: findings from the Whitehall II Study. Sleep 2011;34:565-73.

26 Jelicic M, Bosma $\mathrm{H}$, Ponds RW, et al. Subjective sleep problems in later life as predictors of cognitive decline. Report from the Maastricht Ageing Study (MAAS). Int J Geriatr Psychiatry 2002;17:73-7.

27 Elwood PC, Bayer AJ, Fish M, et al. Sleep disturbance and daytime sleepiness predict vascular dementia. J Epidemio/ Community Health 2011:65:820-4.

28 Sterniczuk R, Theou 0, Rusak B, et al. Sleep disturbance is associated with incident dementia and mortality. Curr Alzheimer Res 2013;10:767-75.

29 Jaussent I, Bouyer J, Ancelin ML, et al. Excessive sleepiness is predictive of cognitive decline in the elderly. Sleep 2012;35:1201-7.

30 Benito-León J, Bermejo-Pareja F, Vega S, et al. Total daily sleep duration and the risk of dementia: a prospective population-based study. Eur J Neurol 2009;16:990-7.

31 Foley D, Monjan A, Masaki K, et al. Daytime sleepiness is associated with 3-year incident dementia and cognitive decline in older Japanese-American men. J Am Geriatr Soc 2001;49:1628-32.

32 Virta JJ, Heikkilä K, Perola M, et al. Midlife sleep characteristics associated with late life cognitive function. Sleep 2013;36:1533-41.

33 Hahn EA, Wang HX, Andel $\mathrm{R}$, et al. A change in sleep pattern May predict Alzheimer disease. Am J Geriatr Psychiatry 2014;22:1262-71.

34 Salonen JT. Is there a continuing need for longitudinal epidemiologic research? The Kuopio ischaemic heart disease risk factor study. Ann Clin Res 1996;41:541-9.

35 National Institute for Health and Welfare. Care Register for Health Care, registe description. Updated: 25 Feb 2016. http://www.thl.fi/en/web/thlfi-en/statistics/ information-on-statistics/register-descriptions/care-register-for-health-care (accessed: 29 Jun 2016).

36 Statistics Finland. Health Statistics: Causes of death, quality descriptions. Updated 30 Dec 2015. http://www.stat.fi/til/ksyyt/laa_en.html (Accessed 29 Jun 2016).

37 Kajantie M, Manderbacka K, McCallum A, et al. How to carry out register-based health services research in Finland. In STAKES Discussion papers 1/2006 http:/l www.stakes.fi/verkkojulkaisut/papers/DP1-2006.pdf (accessed: 29 Jun 2016).

38 Tolppanen AM, Taipale $\mathrm{H}$, Koponen $\mathrm{M}$, et al. Use of existing data sources in clinical epidemiology: Finnish health care registers in Alzheimer's disease research-the Medication use among persons with Alzheimer's disease (MEDALZ-2005) study. Clin Epidemiol 2013:5:277-85.

39 Alander J, Lönnroos E, Hartikainen S, et al. Nationwide use of medicines for Alzheimer's disease by community-dwelling persons in Finland. J Am Geriatr Soc 2006;54:557-8

40 Virta L, Viramo P. Incidence of cholinergic and memantine treatment in Alzheimer's disease in Finland. J Am Geriatr Soc 2007;55:1886-7.

41 American Psychiatric Association. Diagnostic and statistical manual of mental disorders. 4th edn. Washington DC: American Psychiatric Association, 1994.

42 McKhann G, Drachman D, Folstein M, et al. Clinical diagnosis of Alzheimer's disease: report of the NINCDS-ADRDA Work Group under the auspices of Department of Health and Human Services Task Force on Alzheimer's Disease. Neurology 1984;34:939-44.

43 McKhann GM, Knopman DS, Chertkow H, et al. The diagnosis of dementia due to Alzheimer's disease: recommendations from the National Institute on 
Aging-Alzheimer's Association workgroups on diagnostic guidelines for Alzheimer's disease. Alzheimers Dement 2011;7:263-9.

44 Kaplan GA, Roberts RE, Camacho TC, et al. Psychosocial predictors of depression. Prospective evidence from the human population laboratory studies. Am J Epidemiol 1987;125:206-20.

45 Salonen JT, Slater JS, Tuomilehto J, et al. Leisure time and occupational physical activity: risk of death from ischemic heart disease. Am J Epidemiol 1988;127:87-94.

46 Kauhanen J, Julkunen J, Salonen JT. Coping with inner feelings and stress: heavy alcohol use in the context of alexithymia. Behav Med 1992;18:121-6.

47 Sorlie PD, Cooper L, Schreiner PJ, et al. Repeatability and validity of the Rose questionnaire for angina pectoris in the Atherosclerosis Risk in Communities Study. J Clin Epidemiol 1996;49:719-25.

48 Tsukamoto K, Watanabe T, Matsushima T, et al. Determination by PCR-RFLP of apo E genotype in a Japanese population. J Lab Clin Med 1993:121:598-602.

49 Vaz Fragoso CA, Gill TM. Sleep complaints in community-living older persons: a multifactorial geriatric syndrome. J Am Geriatr Soc 2007:55:1853-66.

50 Riemann D, Voderholzer U, Spiegelhalder K, et al. Chronic insomnia and MRI-measured hippocampal volumes: a pilot study. Sleep 2007:30:955-8.

51 Neylan TC, Mueller SG, Wang Z, et al. Insomnia severity is associated with a decreased volume of the CA3/dentate gyrus hippocampal subfield. Biol Psychiatry 2010:68:494-6.
52 Altena E, Vrenken H, Van Der Werf YD, et al. Reduced orbitofrontal and parietal gray matter in chronic insomnia: a voxel-based morphometric study. Biol Psychiatry 2010;67:182-5.

53 Winkelman JW, Benson KL, Buxton OM, et al. Lack of hippocampal volume differences in primary insomnia and good sleeper controls: an MRI volumetric study at 3 Tesla. Sleep Med 2010;11:576-82.

54 Spiegelhalder K, Regen W, Baglioni C, et al. Insomnia does not appear to be associated with substantial structural brain changes. Sleep 2013:36:731-7.

55 Zhu B, Dong Y, Xu Z, et al. Sleep disturbance induces neuroinflammation and impairment of learning and memory. Neurobiol Dis 2012;48:348-55.

56 Meerlo P, Mistlberger RE, Jacobs BL, et al. New neurons in the adult brain: the role of sleep and consequences of sleep loss. Sleep Med Rev 2009;13:187-94.

57 American Academy of Sleep Medicine. International Classification of Sleep Disorders. 3rd ed. Darien, IL: American Academy of Sleep Medicine; 2014

58 Slater G, Steier J. Excessive daytime sleepiness in sleep disorders. J Thorac Dis 2012;4:608-16.

59 Young TB. Epidemiology of daytime sleepiness: definitions, symptomatology, and prevalence. J Clin Psychiatry 2004;65(Suppl 16):12-16.

60 Ward A, Crean S, Mercaldi CJ, et al. Prevalence of apolipoprotein E4 genotype and homozygotes (APOE e4/4) among patients diagnosed with Alzheimer's disease: a systematic review and meta-analysis. Neuroepidemiology 2012;38:1-17. 